\title{
Eficiência nutricional do milho cv. BRS 1030 submetido à omissão de macronutrientes em solução nutritiva
}

\author{
Ancélio Ricardo de Oliveira Gondim ${ }^{1}$, Renato de Mello Prado ${ }^{2}$, Adriana Ursulino Alves ${ }^{3}$, Ivana Machado Fonseca ${ }^{3}$
}

\section{RESUMO}

Em casa de vegetação, desenvolveu-se experimento com delineamento inteiramente casualizado, com sete tratamentos e três repetições, a fim de estudar a influência da omissão de nutrientes nas relações de eficiência (eficiência de absorção, translocação e utilização) de plantas de milho cv. BRS 1030 crescidas em solução nutritiva de Hoagland \& Arnon (1950), completa e com omissão de N, P, K, Ca, Mg e S, durante 30 dias. Após esse período, as plantas foram coletadas, separadas em parte aérea e raiz, secadas e em seguida pesadas e moídas, para determinação dos teores de macronutrientes e obtenção dos índices de eficiência. A omissão de macronutrientes promove diminuição na produção de massa seca da planta inteira e na eficiência de absorção dos nutrientes, quando comparada à do tratamento completo. A omissão de P, Mg e S resulta em maior eficiência de utilização, comparada com o tratamento completo. Plantas deficientes retranslocam os nutrientes, tentando manter seu conteúdo nas folhas medianas (ou parte aérea) em relação aos demais órgãos.

Palavras- chave: Zea mays L., cultivo hidropônico, nutrição mineral.

\section{ABSTRACT \\ Uptake and utilization efficiency of corn cultivated in nutrient solution with omission of macronutrients}

The objective of this study was to evaluate the effect of nutrient omission on macronutrient uptake, translocation and utilization efficiency in corn. To achieve this objective a greenhouse experiment was conducted in completely randomized design, with seven treatments and three replications. Corn plants were cultivated in Hoagland \& Arnon (1950) solution complete or with omission of N, P, K, Ca, Mg and S for 30 days. After this period, plants were harvested and separated into aerial parts (leaves+stem) and roots. The plant parts were dried, weighed and ground for the macronutrient determination and calculation of efficiency indices. The omission of macronutrients reduced the dry mass of the whole plant and uptake efficiency compared with the complete solution. The omission of $\mathrm{P}, \mathrm{Mg}$ and $\mathrm{S}$ resulted in higher utilization efficiency compared with the complete solution. In deficient plants, nutrients are retranslocated from the other organs to middle leaves of the plant in order to maintain its contents.

Key words: Hydroponic cultivation, mineral nutrition, Zea mays L.

\footnotetext{
Recebido para publicação em novembro de 2008 e aprovado em julho de 2010

${ }^{1}$ Engenheiro Agrônomo, Doutor. Instituto Federal de Educação, Ciência e Tecnologia do Ceará,. BR 110, Km 47, Vila cajazeiras, 63500-000, Iguatu, Ceará, Brasil. anceliogondim@hotmail.com

${ }^{2}$ Engenheiro Agrônomo, Doutor. Universidade Estadual Paulista Júlio de Mesquita Filho, Faculdade de Ciências Agrárias e Veterinárias de Jaboticabal, Departamento de Solos e Adubos, Via de Acesso Prof. Paulo Donato Castellane, s/n, 14884-900, Jaboticabal, São Paulo, Brasil. rmprado@fcav.unesp.br

Engenheiro Agrônomo, Mestre. Universidade Estadual Paulista Júlio de Mesquita Filho, Faculdade de Ciências Agrárias e Veterinárias de Jaboticabal, Departamento de Solos e Adubos, Via de Acesso Prof. Paulo Donato Castellane, s/n, 14884-900, Jaboticabal, São Paulo, Brasil.
} 


\section{INTRODUÇÃO}

O milho (Zea mays) é a mais importante cultura de grãos do mundo. É reconhecido como cereal vital, de alta produtividade e de uso diversificado (Islam \& Kaul, 1986) e apresenta alto conteúdo de proteínas, acima de outros cereais como, por exemplo, o arroz (Alam et al., 2003).

O aproveitamento da absorção e utilização de nutrientes, visando a maximizar a eficiência no uso de nutrientes e, portanto, otimizar a produtividade das culturas, tem sido bastante estudado nos últimos anos (Elliot \& Lauchli, 1985).

As culturas são afetadas diretamente pela eficiência nutricional, que indica a capacidade da planta em extrair nutrientes do meio de cultivo. Sabe-se que os mecanismos que governam a eficiência de absorção, que indica a capacidade da planta em converter o nutriente absorvido em matéria seca, diferem entre as espécies, já que algumas apresentam sistema radicular extenso, outras apresentam elevada taxa de absorção por unidade de comprimento de raiz (Föhse et al., 1998). Já a eficiência de uso é a capacidade de uma planta redistribuir e reutilizar os elementos minerais de um órgão mais velho e senescente (Gerloff \& Gabelman, 1983). Lauchli (1987) complementa que a eficiência de uso de um nutriente é definida como a relação entre a concentração do nutriente no tecido e a produção.

Machado (2000) observou que plantas submetidas a estresse de fósforo (P) apresentam inibição no crescimento total e aumento relativo no sistema radicular, significando que a redução no crescimento da parte aérea acontece antes da redução no crescimento das raízes, ou seja, como o sistema radicular está mais próximo do $\mathrm{P}$, seu crescimento ainda permanece por determinado período.

O cultivo de plantas em vasos, utilizando solução nutritiva, é ferramenta muito útil nos estudos de nutrição mineral e na busca de soluções e de genótipos mais eficientes. Com a melhoria da eficiência nutricional, aumentase a produtividade e reduz-se o emprego de adubos, com consequente diminuição nos custos. Assim, a otimização da eficiência nutricional é de grande importância, pois os fertilizantes contribuem com aproximadamente $30 \%$ do custo total da produção (Fageria, 1998).

Rosolem \& Marcello (1998), estudando a influência de níveis de Ca e P no crescimento radicular da soja cultivar IAC 17, observaram que houve formação de raízes mais finas e alongadas, em resposta à baixa disponibilidade de P. Teruel et al. (2001) constataram que plantas cultivadas em substrato com omissão de $\mathrm{P}$ apresentaram sistema radicular com maior número de raízes e mais profundo, sendo consideradas estratégias adaptativas das plantas à falta do nutriente.

A deficiência nutricional poderá causar diminuição no crescimento das plantas em decorrência de alterações na eficiência nutricional, constituindo aspecto pou- co abordado na literatura que trata da omissão de nutrientes em plantas.

Assim, o objetivo deste trabalho foi estudar o efeito da omissão de macronutrientes na eficiência nutricional do cultivar de milho BRS 1030 em solução nutritiva.

\section{MATERIAL E MÉTODOS}

O experimento foi conduzido em casa de vegetação, sob hidroponia, na FCAV/Unesp, campus Jaboticabal-SP.

Sementes de milho cv. BRS 1030, previamente desinfetadas em hipoclorito de sódio a 1\%, durante 5 minutos, e lavadas em água destilada, foram colocadas para germinar em tubetes ( $15 \mathrm{~cm}$ de profundidade), preenchidos com substrato à base de casca de pinus e vermiculita, na proporção 1:1 (v/v), e foram irrigadas com água até a emergência; em seguida, foi aplicada solução nutritiva de Hoagland \& Arnon (1950) diluída a 25\%, durante a fase de crescimento inicial até duas semanas após a emergência.

Após esse período, duas plântulas, previamente selecionadas quanto à sanidade e uniformidade, foram transferidas para cada vaso, contendo 2,5 L de solução nutritiva de cada tratamento. A partir deste momento, as plantas foram cultivadas nas diversas soluções nutritivas, com omissão de macronutriente que teve como base a solução nutritiva de Hoagland \& Arnon (1950), contendo as seguintes concentrações de nutrientes, em $\mathrm{mg} \mathrm{L}^{-1}: 210,1$ de N; 31,0 de P; 234,6 de K; 200,4 de Ca; 48,6 de Mg; 64,2 de S; 0,5 de B; 0,02 de Cu; 0,6 de Cl; 5 de Fe; 0,5 de Mn; 0,01 de Mo e 0,05 de Zn.

A solução nutritiva foi aerada diariamente e o $\mathrm{pH}$ ajustado entre 5,0 e 6,0, usando-se, respectivamente, $\mathrm{HCl}$ e $\mathrm{NaOH}$ a $0,5 \mathrm{~mol} \mathrm{~L}^{-1,}$ quando esta se encontrava acima ou abaixo desta faixa. O volume de solução nutritiva do vaso foi mantido constante, acrescentando-se, diariamente, água deionizada. Aos 15 dias, fez-se a substituição da solução nutritiva por uma nova.

O delineamento experimental foi o inteiramente casualizado, com sete tratamentos (completo e -N, -P, -K, -Ca, - Mg e -S) e três repetições. A unidade experimental foi constituída por um vaso contendo duas plantas.

Os sintomas de deficiência foram observados desde o início, com aparecimento das primeiras desordens nutricionais. Quando o sintoma de deficiência de determinado nutriente estava muito bem definido, fez-se a colheita das plantas do tratamento correspondente. Nessas ocasiões, foram avaliados o diâmetro do caule $(2,0 \mathrm{~cm}$ do colo); o número de folhas por planta; a altura da planta (colo da planta até a folha mais alta) e a área foliar.

Após 30 dias da aplicação dos tratamentos, quando as plantas apresentavam em média sete folhas completamente expandidas no tratamento sem omissão, procedeuse à colheita das plantas, que foram submetidas à lava- 
gem em solução de detergente $\left(3 \mathrm{ml} \mathrm{L}^{-1}\right)$, água corrente, solução de $\mathrm{HCl} 0,1 \mathrm{~mol} \mathrm{~L}^{-1}\left(8,4 \mathrm{ml} \mathrm{L}^{-1}\right)$ e água destilada. Foram separadas a parte aérea e raízes e colocadas para secar em estufa com circulação forçada de ar a $70^{\circ} \mathrm{C}$ até massa constante. Obtida a matéria seca da parte aérea e das raízes, procedeu-se à moagem do material em moinho tipo Willey, para análise química de tecido vegetal. Foram determinados os teores de N, P, K, Ca, Mg e S na parte aérea e na raiz, segundo metodologias descritas por Bataglia et al. (1983).

A partir da matéria seca e do conteúdo dos nutrientes na planta, foram calculados os índices: (a) eficiência de absorção $(E A B)=($ acúmulo parte aérea + raiz $) /($ matéria seca de raízes), conforme Swiader et al. (1994); (b) eficiência de transporte $(E T)=(($ acúmulo do nutriente na parte aérea)/( acúmulo parte aérea + raiz)) x 100, de acordo com Li et al. (1991) e (c) eficiência de utilização (EU) = [(massa seca total) $2 /($ acúmulo parte aérea + raiz)], segundo Siddiqi \& Glass (1981).

Os dados obtidos foram submetidos à análise de variância (teste F) pelo "software” SAEG 8.0 (1999). Quando significativos, as médias foram comparadas pelo teste de Tukey a 5\% de probabilidade.

\section{RESULTADOS E DISCUSSÃO}

Para todas as variáveis avaliadas houve efeito significativo dos tratamentos.

O diâmetro, a altura, o número de folhas e a área foliar das plantas de milho encontram-se na Tabela 1. A omissão de $\mathrm{N}$ foi o tratamento que mais limitou o crescimento inicial do milho durante o experimento, pois, observou-se que o diâmetro, altura, o número de folhas e a área foliar foram inferiores quando comparados aos do tratamento sem omissão. A restrição de $\mathrm{N}$ levou a uma redução de crescimento, pois, conforme Marschner (1995) e Malavolta et al. (1997), esse nutriente, além de fazer parte da estrutura de aminoácidos, proteínas, bases nitrogenadas, ácidos nucleicos, enzimas, coenzimas, vitaminas, pigmentos e produtos secundários, participa de processos como absorção iônica, fotossíntese, respiração, multiplicação e diferenciação celular, os quais interferem direta ou indiretamente no desenvolvimento da planta.

A produção de matéria seca da parte aérea, raiz e planta inteira é apresentada na Tabela 2. A omissão de N, P, K, $\mathrm{Ca}, \mathrm{Mg}$ ou $\mathrm{S}$ na solução nutritiva levaram ao decréscimo de matéria seca na parte aérea, raízes e planta inteira de plantas de milho cultivadas sob hidroponia. Portanto, como era esperado, o tratamento sem omissão de nutrientes apresentou maior acúmulo de matéria seca que os demais tratamentos com omissão de nutrientes. A omissão de $\mathrm{N}$ foi o fator mais limitante na produção inicial de matéria seca da parte aérea e da planta inteira, diferindo significativamente do tratamento sem omissão de nutrientes. Espécies de crescimento inicial rápido como o milho exigem grande quantidade de $\mathrm{N}$ em curto intervalo de tempo e o não suprimento resultará em prejuízo no crescimento inicial (Almeida et al., 2003).

A matéria seca da parte aérea foi reduzida pela omissão de N, K, P, Ca, S ou Mg (Tabela 2) e todas diferiram estatisticamente do tratamento completo.

A matéria seca das raízes foi reduzida pela omissão de $\mathrm{K}, \mathrm{Mg}, \mathrm{N}, \mathrm{Ca}, \mathrm{S}$ ou P, em ordem de intensidade do efeito da omissão de cada nutriente, também diferindo estatisticamente do tratamento completo (Tabela 2).

Observou-se que os tratamentos diferiram significativamente em relação à eficiência de absorção (EA) dos macronutrientes. Plantas adequadamente nutridas apresentaram maior EA, ocorrendo o contrário para plantas deficientes (Tabela 3).

O nitrogênio e o potássio foram os nutrientes mais requeridos na fase inicial de desenvolvimento do milho. É conhecida a importância do nitrogênio como constituinte essencial dos aminoácidos, principais integrantes de proteínas, além da sua atuação na divisão celular e na produção de clorofila. Por sua vez, o potássio atua em processos osmóticos, na síntese de proteínas, na manutenção

Tabela 1. Diâmetro do caule (DC), altura (ALT), número de folha (NF) e área foliar (AF), de planta de milho cultivada em soluções nutritivas completas e com omissão de macronutriente

\begin{tabular}{|c|c|c|c|c|}
\hline Tratamentos & $\begin{array}{l}\mathrm{DC} \\
\mathrm{mm}\end{array}$ & $\begin{array}{l}\text { ALT } \\
\text { cm }\end{array}$ & NF & $\begin{array}{c}\text { AF } \\
\mathbf{c m}^{2} / \text { planta }\end{array}$ \\
\hline *Completo & $9,5 \mathrm{a}$ & $75,8 \mathrm{a}$ & $5,0 \mathrm{a}$ & 1766,4 a \\
\hline$-\mathrm{N}$ & $6,1 \mathrm{c}$ & $50,0 \mathrm{~d}$ & $3,0 \mathrm{~b}$ & $285,1 \mathrm{~d}$ \\
\hline$-\mathrm{P}$ & $8,4 \mathrm{~b}$ & 69,2 c & $3,0 \mathrm{~b}$ & $832,8 \mathrm{~b}$ \\
\hline$-K$ & $7,8 \mathrm{~b}$ & $62,2 \mathrm{c}$ & $5,0 \mathrm{a}$ & 652,7 c \\
\hline$-\mathrm{Ca}$ & 8,9 a & $60,9 \mathrm{~b}$ & $4,0 \mathrm{a}$ & $795,0 \mathrm{~b}$ \\
\hline$-\mathrm{Mg}$ & 8,9 a & $69,4 \mathrm{a}$ & $4,0 \mathrm{a}$ & 1606,3 a \\
\hline$-S$ & $9,1 \mathrm{a}$ & 66,0 a & $4,0 \mathrm{a}$ & 1337,2 a \\
\hline$\overline{\mathrm{CV} \%}$ & 11,3 & 10,7 & 9,3 & 4,8 \\
\hline
\end{tabular}

Médias seguidas de mesma letra na coluna não diferem a 5\% de probabilidade pelo teste de Tukey. * completo: sem omissão de nutrientes (solução de Hoagland \& Arnon (1950)). 
Tabela 2. Produção de matéria seca da parte aérea, raízes e planta inteira de planta de milho cultivada em soluções nutritivas completas e com omissão de macronutriente

\begin{tabular}{lccc}
\hline \multirow{2}{*}{ Tratamento } & & Matéria seca & Planta inteira \\
\cline { 2 - 4 } & Parte aérea & Raiz & g/planta \\
*Completo & $15,60 \mathrm{a}$ & $3,14 \mathrm{a}$ & $2,74 \mathrm{a}$ \\
- N & $1,76 \mathrm{c}$ & $1,13 \mathrm{bc}$ & $5,89 \mathrm{bc}$ \\
- P & $4,04 \mathrm{bc}$ & $1,85 \mathrm{~b}$ & $3,76 \mathrm{bc}$ \\
- K & $3,04 \mathrm{bc}$ & $0,72 \mathrm{c}$ & $5,25 \mathrm{bc}$ \\
- Ca & $4,05 \mathrm{bc}$ & $1,20 \mathrm{bc}$ & $7,99 \mathrm{bc}$ \\
- Mg & $6,91 \mathrm{~b}$ & $1,08 \mathrm{bc}$ & $8,01 \mathrm{~b}$ \\
\hline S & $6,60 \mathrm{~b}$ & $1,41 \mathrm{bc}$ & 24,4 \\
\hline CV $(\%)$ & 24,3 & 25,6 & $\mathrm{C}$
\end{tabular}

As médias seguidas da mesma letra minúscula na vertical, não diferem estatisticamente entre si, pelo teste Tukey a 5\% de probabilidade.

* completo: sem omissão de nutrientes (solução de Hoagland \& Arnon (1950)).

Tabela 3. Eficiência de absorção de nutrientes de plantas de milho, sob cultivo em soluções nutritivas completas e com omissão de macronutrientes

\begin{tabular}{lcccccc}
\hline Tratamentos & $\mathbf{N}$ & $\mathbf{P}$ & $\mathbf{K}$ & $\mathbf{C a}$ & $\mathbf{M g}$ & $\mathbf{S}$ \\
\hline & & & $\boldsymbol{m g}$ nutriente / $\boldsymbol{g}$ matéria seca raízes & & \\
*Completo & $325,7 \mathrm{~b}$ & $62,2 \mathrm{c}$ & $564,0 \mathrm{~b}$ & $78,3 \mathrm{bc}$ & $69,3 \mathrm{a}$ & $51,3 \mathrm{a}$ \\
- N & $36,9 \mathrm{~d}$ & $47,8 \mathrm{c}$ & $206,6 \mathrm{de}$ & $22,3 \mathrm{~d}$ & $11,6 \mathrm{c}$ & $10,4 \mathrm{c}$ \\
- P & $150,1 \mathrm{c}$ & $6,0 \mathrm{~d}$ & $343,3 \mathrm{c}$ & $31,6 \mathrm{~cd}$ & $16,6 \mathrm{c}$ & $18,6 \mathrm{c}$ \\
- K & $447,2 \mathrm{a}$ & $103,2 \mathrm{ab}$ & $114,9 \mathrm{e}$ & $135,2 \mathrm{a}$ & $49,9 \mathrm{~b}$ & $41,5 \mathrm{ab}$ \\
- Ca & $379,3 \mathrm{ab}$ & $76,3 \mathrm{bc}$ & $347,8 \mathrm{~cd}$ & $12,9 \mathrm{~d}$ & $43,8 \mathrm{~b}$ & $46,1 \mathrm{a}$ \\
- Mg & $435,3 \mathrm{a}$ & $125,1 \mathrm{a}$ & $812,6 \mathrm{a}$ & $118,6 \mathrm{ab}$ & $12,0 \mathrm{c}$ & $43,6 \mathrm{a}$ \\
- S & $324,4 \mathrm{~b}$ & $108,1 \mathrm{ab}$ & $682,4 \mathrm{ab}$ & $85,2 \mathrm{abc}$ & $48,5 \mathrm{~b}$ & $22,2 \mathrm{bc}$ \\
\hline CV (\%) & 11,5 & 18,3 & 12,0 & 28,0 & 9,8 & 22,5 \\
\hline
\end{tabular}

As médias seguidas da mesma letra minúscula na vertical, não diferem estatisticamente entre si, pelo teste Tukey a 5\% de probabilidade.

* completo: sem omissão de nutrientes (solução de Hoagland \& Arnon (1950)).

da estabilidade e permeabilidade das membranas e no controle do pH (Malavolta et al., 1997; Marschner, 1995). Essas funções de ambos os nutrientes podem explicar a exigência da planta, que se encontra em intensa atividade metabólica nesta fase inicial de desenvolvimento.

Certamente, a menor EA apresentada pelas plantas deficientes ocorreu porque a sua absorção foi menor não por ineficiência do sistema de absorção, mas, pela baixa disponibilidade do nutriente.

Quanto à eficiência de transporte (ET), verificou-se que o K foi o nutriente que obteve os maiores índices, independentemente do tratamento empregado (Tabela 4). A eficiência de transporte é controlada pela capacidade do solo ou sistema de cultivo em suprir os nutrientes e pela capacidade das plantas para absorver, utilizar, e remover os nutrientes. Esses fatores variam com solos, tipo de genótipo/cultivares de várias espécies, bem como com as condições climáticas, sendo que envolvem um sincronismo no sistema solo-planta-raiz (Baligar \& Fageria, 1997). Relatos diferentes são disponíveis em tomate (Behling et al., 1989) e carambola (Rozane et al., 2007), para os quais o nutriente que apresentou maior eficiência de transporte foi o cálcio, fato este atribuído às altas concentrações desse nutriente insolúvel nos tecidos da parte aérea da planta.

Os tratamentos diferiram quanto à ET dos macronutrientes, exceto em relação ao potássio. As omissões de P e S apresentaram maior eficiência de transporte para o nitrogênio, em relação aos demais tratamentos. Contudo, o tratamento com omissão de $\mathrm{N}$ apresentou menor eficiência de transporte para o fósforo, em relação aos demais tratamentos. A maior eficiência de transporte para Ca foi observada nos tratamentos com omissão de N e P. Já a maior eficiência de transporte para Mg foi observada nos tratamentos com omissão de N, P, K e Ca e os tratamentos com omissão de P, $\mathrm{K}$, Ca Mg e S foram os que apresentaram maior eficiência de transporte para o enxofre (Tabela 4).

Com exceção da omissão de $\mathrm{N}$, todos os tratamentos apresentaram alta eficiência de utilização (EU) quando cada nutriente foi omitido (Tabela 5). A EU do P variou de 154,27 a 3235,83 $\mathrm{g} \mathrm{mg}^{-1}$ (Tabela 5). O menor valor (154,27 $\mathrm{g} \mathrm{mg}^{-1}$ ) foi observado em plantas no tratamento com omissão de $\mathrm{N}$.

O valor da EU mostrou que plantas deficientes em $\mathrm{K}$, P, Ca, Mg e S foram mais eficazes, produzindo mais matéria seca por unidade de mineral absorvido (Tabela 5). Como 
no presente trabalho ofereceram-se condições de estresse fisiológico ou falta de nutrientes, para as plantas, era de se esperar que houvesse grandes diferenças entre os tratamentos na eficiência de utilização.

Nota-se maior eficiência de utilização de P, Ca, Mg e S nos tratamentos com omissão destes nutrientes, com- parando-se com o tratamento completo, podendo-se observar que os tratamentos com omissão de P, Ca, Mg e S apresentaram valores médios de 3235,83; 1757,75; 5112,04 e 3277,66, superiores aos apresentados no tratamento completo para os mesmos nutrientes, de 1813,05; 1431,92; 1620,64 e 2194,08, respectivamente (Tabela 5).

Tabela 4. Eficiência de transporte de nutrientes de plantas de milho, sob cultivo em soluções nutritivas completas e com omissão de macronutrientes

\begin{tabular}{lcccccc}
\hline Tratamentos & $\mathbf{N}$ & $\mathbf{P}$ & $\mathbf{K}$ & $\mathbf{C a}$ & $\mathbf{M g}$ & $\mathbf{S}$ \\
\hline & & & $\mathbf{2}$ & & \\
*Completo & $56,1 \mathrm{bc}$ & $56,7 \mathrm{ab}$ & $67,4 \mathrm{a}$ & $46,2 \mathrm{c}$ & $39,2 \mathrm{~b}$ & $20,9 \mathrm{~b}$ \\
- N & $53,4 \mathrm{bc}$ & $42,9 \mathrm{~b}$ & $60,2 \mathrm{a}$ & $84,5 \mathrm{a}$ & $70,9 \mathrm{a}$ & $22,5 \mathrm{~b}$ \\
- P & $64,1 \mathrm{a}$ & $64,2 \mathrm{a}$ & $71,5 \mathrm{a}$ & $73,3 \mathrm{ab}$ & $71,0 \mathrm{a}$ & $28,7 \mathrm{ab}$ \\
- K & $51,7 \mathrm{bc}$ & $52,8 \mathrm{ab}$ & $72,2 \mathrm{a}$ & $50,1 \mathrm{c}$ & $74,5 \mathrm{a}$ & $35,8 \mathrm{ab}$ \\
- Ca & $50,3 \mathrm{c}$ & $49,0 \mathrm{ab}$ & $60,2 \mathrm{a}$ & $59,6 \mathrm{bc}$ & $68,8 \mathrm{a}$ & $34,0 \mathrm{ab}$ \\
- Mg & $54,9 \mathrm{bc}$ & $51,0 \mathrm{ab}$ & $65,1 \mathrm{a}$ & $38,6 \mathrm{c}$ & $45,5 \mathrm{~b}$ & $26,2 \mathrm{~b}$ \\
- S & $58,5 \mathrm{ab}$ & $49,2 \mathrm{ab}$ & $66,9 \mathrm{a}$ & $51,0 \mathrm{bc}$ & $41,9 \mathrm{~b}$ & $46,5 \mathrm{a}$ \\
\hline CV (\%) & 4,6 & 12,3 & 6,6 & 14,3 & 8,2 & 25,4 \\
\hline
\end{tabular}

As médias seguidas da mesma letra minúscula na vertical, não diferem estatisticamente entre si, pelo teste Tukey a $5 \%$ de probabilidade.

* completo: sem omissão de nutrientes (solução de Hoagland \& Arnon (1950)).

Tabela 5. Eficiência de utilização de nutrientes de plantas de milho, sob cultivo em soluções nutritivas completas e com omissão de macronutrientes

\begin{tabular}{|c|c|c|c|c|c|c|}
\hline Tratamentos & $\mathbf{N}$ & $\mathbf{P}$ & $\mathbf{K}$ & Ca & Mg & $\mathbf{S}$ \\
\hline & \multicolumn{6}{|c|}{ (massa seca total$\left.{ }^{2}\right) \mathrm{g} / \mathrm{mg}$ acúmulo da planta inteira parte aérea + raiz) } \\
\hline *Completo & $344,5 a$ & $1813,0 \mathrm{~b}$ & $198,8 \mathrm{a}$ & $1431,9 a$ & $1620,6 b$ & $2194,1 b$ \\
\hline$-\mathrm{N}$ & $199,6 b$ & $154,3 c$ & $35,9 b$ & $337,4 b$ & $645,5 \mathrm{~cd}$ & $702,5 \mathrm{~cd}$ \\
\hline$-\mathrm{P}$ & $126,3 \mathrm{c}$ & $3235,8 a$ & $51,0 \mathrm{~b}$ & $602,3 b$ & $1117,7 \mathrm{bc}$ & $1024,7 \mathrm{~cd}$ \\
\hline$-\mathrm{K}$ & $45,5 d$ & $205,9 c$ & $179,0 \mathrm{a}$ & $174,2 b$ & 398,3d & 490,3d \\
\hline - $\mathrm{Ca}$ & $58,5 d$ & $283,9 \mathrm{c}$ & $66,3 b$ & $1757,7 a$ & $519,0 \mathrm{~cd}$ & $483,5 d$ \\
\hline - Mg & $136,2 \mathrm{c}$ & $480,1 \mathrm{c}$ & $72,9 b$ & $502,1 b$ & 5112,0a & $1364,5 c$ \\
\hline$-S$ & $141,7 \mathrm{c}$ & $441,9 \mathrm{c}$ & $67,4 b$ & $553,9 b$ & $959,2 \mathrm{~cd}$ & $3277,7 a$ \\
\hline$\overline{\mathrm{CV}(\%)}$ & 7,2 & 14,5 & 24,5 & 25,5 & 15,0 & 20,3 \\
\hline
\end{tabular}

As médias seguidas da mesma letra minúscula na vertical, não diferem estatisticamente entre si, pelo teste Tukey a 5\% de probabilidade.

* completo: sem omissão de nutrientes (solução de Hoagland \& Arnon (1950)).

\section{CONCLUSÕES}

A omissão de macronutrientes na nutrição de plantas de milho promove diminuição na produção de massa seca da parte aérea e na eficiência de absorção de nutrientes.

A omissão de P, Ca, Mg e S na nutrição de plantas de milho resulta em maior eficiência de utilização, comparado com as plantas nutridas adequadamente.

Plantas deficientes retranslocam os nutrientes, tentando manter seu conteúdo na parte aérea em relação aos demais órgãos.

\section{REFERÊNCIAS}

Alam MM, Basher MDM, Karim A, Rahman MA \& Islam MR (2003) Effect of rate of nitrogen fertilizer and population density on the yield and yield attributes of maize (Zea mays L.). Pakistan journal of biological sciences, 6:1770-1773.
Almeida ML, Sangoi L, Nava IC, Galio J, Trentin OS \& Rampazzo C (2003) Crescimento inicial de milho e sua relação com o rendimento de grãos. Ciência Rural, 33:189-194.

Bataglia OC, Furlani AMC, Teixeira JPF, Furlani PR \& Gallo JR (1983) Métodos de análise química de plantas. Campinas, Instituto Agronômico (Boletim Técnico, 78). 48p.

Baligar VC \& Fageria NK (1997) Nutrient use efficiency in acid soils: nutrient management and plant use efficiency. In: $4^{\circ}$ International Symposium on Plant-Soil interactions at low $\mathrm{pH}$, Belo Horizonte. Proceedings, Brazilian Soil Science Society. p.76-95.

Behling JP, Gabelman WH \& Gerloff GC (1989) The distribution and utilization of calcium by two tomato (Lycopersicon esculentum Mill.) lines differing in calcium efficiency when grown under low-Ca stress. Plant Soil, 113:189-196.

Elliot GC \& Lauchli A (1985) Phosphorus efficiency and phosphateiron interaction in maize. Agronomy Journal, 77:399-403.

Fageria NK (1998) Otimização da eficiência nutricional na produção das culturas. Revista Brasileira de Engenharia Agrícola e Ambiental, 2:6-16.

Rev. Ceres, Viçosa, v. 57, n.4, p. 539-544, jul/ago, 2010 
Fohse D, Claassen N \& Jungk A (1998) Phosphorus efficiency of plants: External and internal $\mathrm{P}$ requirement and $\mathrm{P}$ uptake efficiency of different plant species. Plant and Soil, 110:101109.

Gerloff GC \& Gabelman WH (1983) Genetics basis of inorganic plant nutrition. In: Lauchli A \& Bieleski RL (eds). Inorganic plant nutrition. New York, Springer-Verlag, p.453-480.

Hoagland DR \& Arnon DI (1950) The water culture method for growing plants without soils. Berkeley, California Agricultural Experimental Station. 347p.

Islam TMT \& Kaul AK (1986) Prospects of maize in Bangladesh. Bangladesh, FAO/UNDP-Publication Dhaka. 225p.

Lauchli A (1987) Soil science in the next twenty five years: does a biotechnology play a role? Soil Science Society of American Journal, 51:1405-1409.

Li B, Mckeand SE \& Allen HL (1991) Genetic variation in nitrogen use efficiency of loblolly pine seedlings. Forest Science, 37:613626.

Machado CTT (2000) Caracterização de genótipos de milho quanto a parâmetros morfológicos, fisiológicos e microbiológicos associados à eficiência de absorção e uso de fósforo. Tese de doutorado. Universidade Federal Rural do Rio de Janeiro, Rio de Janeiro. 366p.
Malavolta E, Vitti GC \& Oliveira AS (1997) Avaliação do estado nutricional das plantas: princípios e aplicações. 2. ed. Piracicaba, POTAFOS. 319p.

Marschner H (1995) Mineral nutrition of higher plants. London, Academic. 889p.

Rosolem CA \& Marcello CS (1998) Crescimento radicular e nutrição mineral da soja em função da calagem e adubação fosfatada. Scientia Agrícola, 55:448-455.

Rozane DE, Prado RM, Franco CF \& Natale W (2007) Eficiência de absorção, transporte e utilização de macronutrientes por porta-enxertos de caramboleira, cultivados em soluções nutritivas. Ciência agrotecnologia, 31:1020-1026.

SAEG - Sistema para análises estatísticas (1999) versão 8.0. Universidade Federal de Viçosa, MG.

Siddiqi MY \& Glass ADM (1981) Utilization index: a modified approach to the estimation and comparison of nutrient efficiency in plants. Journal of Plant Nutrition, 4:289-302.

Swiader JM, Chyan Y \& Freiji FG (1994) Genotypic differences in nitrate uptake and utilization efficiency in pumpkin hybrids. Journal of Plant Nutrition, 17:1687-1699.

Teruel DA, Dourado Neto D, Hopmans JW \& Reichardt K (2001) Alterações estruturais do sistema radicular de soja em resposta à disponibilidade de fósforo no solo. Scientia Agrícola, 58:55-60. 